hep-ph/9504265

\title{
Particle Currents on a CP Violating Higgs Background and the Spontaneous Baryogenesis Mechanism
}

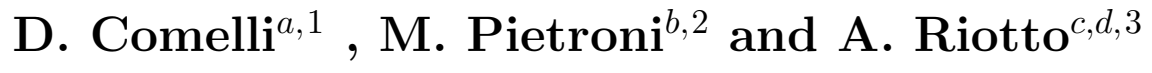 \\ (a) Departamento de Fisica Teorica, Universitad de Valencia, \\ E-46110 Burjassot, Valencia, Spain \\ (b) Deutsches Elektronen-Synchrotron DESY, \\ Notkestr. 85, D-22603 Hamburg, Germany. \\ ${ }^{(c)}$ Istituto Nazionale di Fisica Nucleare, \\ Sezione di Padova, 35100 Padua, Italy. \\ (d) International School for Advanced Studies, SISSA-ISAS \\ Strada Costiera 11, I-34014 Miramare, Trieste, Italy.
}

\begin{abstract}
We compute the particle currents induced on a bubble wall background at finite temperature in a model with $\mathrm{CP}$ violation in the Higgs sector. Using a field theory approach we show that fermionic currents arise at one loop, so that a suppression factor $\mathcal{O}\left(h_{t} \phi / \pi T\right)^{2}$ with respect to previous computations is found. The contributions to the Higgs currents are also derived and their relevancy for the spontaneous baryogenesis mechanism is discussed.
\end{abstract}

\footnotetext{
${ }^{1}$ Email: comelli@evalvx.ific.uv.es. Work supported by Ministerio de Educacion y Ciencia de España

${ }^{2}$ Email: pietroni@vxdesy.desy.de

${ }^{3}$ Email:riotto@tsmi19.sissa.it. Permanent address after November 95: Theoretical Astrophysics Group, NASA/Fermilab, Batavia, Il 60510
} 
The possibility of generating the baryon asymmetry of the Universe during the electroweak phase transition has received much attention in the last years [1, 21. In the usual scenarios the transition is required to be first order and to proceed via nucleation of bubbles of the broken phase in the unbroken phase. The necessary departure from thermal equilibrium then can take place inside or in front of the walls of the expanding bubbles.

Two different limits have been investigated in the literature. In the case of thin bubble walls, the asymmetric (in fermion numbers) reflection of particles off the bubble wall is the dominant effect. The induced fermion number flux is then reprocessed into a baryon asymmetry by the anomalous, $(B+L)$-violating, sphaleronic transitions in the unbroken phase [3].

In this letter we will focus on the opposite limit of thick bubble walls. Indeed, this is thought to be the relevant one if the phase transition is weakly of the first order, as it seems to be the case for the standard model [4, 5] and the minimal supersymmetric extension of it (MSSM) [6]. If the only source of $\mathrm{CP}$ violation is the phase in the Cabibbo-Kobayashi- Maskawa matrix, it seems very hard to generate any baryon asymmetry in this limit. On the other hand, if CP violation is present in the Higgs sector (which requires at least two Higgs doublets), the so called spontaneous baryogenesis mechanism can be invoked [7]. Both in the case of explicit [7] and spontaneous [8] CP violation, a space-time dependent relative phase $\delta(x)$ between the two Higgs fields is turned on inside the bubble wall. In order to analyze the effect of this complex space-time dependent background on particle densities, a rotation on the fields can be performed to make the Yukawa couplings real. As a consequence, a derivative coupling of the form

$$
\mathcal{L}_{\text {int }} \sim \partial_{\mu} \delta J^{\mu},
$$

where $J^{\mu}$ is the current corresponding to the rotation凹, is induced from the kinetic terms.

In the original paper [7], only the time derivative of the phase $\delta$ was taken into account. In this approximation, $\dot{\delta}$ acts as an effective chemical potential (usually called 'charge potential'), and the particle densities are perturbed to nonzero values $n_{i} \sim q_{i} \dot{\delta} T^{2}$, where $q_{i}$ is the charge of the $i$-th particle under the given rotation. In presence of such a charge potential, and of baryon number violation, the thermodynamical evolution of the system adiabatically leads to a non-vanishing baryon asymmetry. This approximation has been improved in ref. [9], where also the spatial derivatives of $\delta$ in eq. (1) were taken into account, and the role of particle

\footnotetext{
${ }^{1}$ In order to avoid anomalies, in the original paper [7] and in [9] the rotation was taken to be the hypercharge. In fact, this can only be done if the same higgs doublet couples both to up and down type quarks. In the models in which two different Higgs doublets are coupled to the up and down type quarks (as is the case for the MSSM) it is not possible to remove the relative phase $\delta$ from all the Yukawa couplings by making a hypercharge rotation [10.
} 
diffusion was discussed.

Nevertheless, we believe that using the interaction term in eq. (11) as a starting point to compute the perturbations to the thermal averages presents some problems.

First of all, the proportionality between an individual particle density and its hypercharge is a direct consequence of the hypercharge rotation made to make the Yukawa couplings real. Making a different rotation, the proportionality would of course drop. For instance, one could rotate the right handed top and leave the left handed one untouched, absorbing the anomaly by a proper rotation of the light fermion fields. In this case, the perturbations to be put in the system of kinetic equations describing the system would be different, so that in principle the final value for the baryon asymmetry might come out to be dependent on the rotation that has been made.

Secondly, since the phase $\delta$ is communicated from the Higgs to the fermion sector through the Yukawa interactions, any perturbation in the fermion densities $n_{i}$ should vanish in the limit of zero Yukawa couplings $h_{i}$. Also, they should vanish in the limit of zero vacuum expectation value for the Higgs fields $H_{i}(x)=v_{i}(x) \exp \left[i \theta_{i}(x)\right]$ because no spontaneous $\mathrm{CP}$ violation is present in the Higgs sector in this limit. Naively, one could then expect a suppression factor of order $\left(h_{i}^{2} v_{i}^{2}(x) / T^{2}\right)$, where $h_{i}$ is the relevant Yukawa coupling, for the perturbations in the fermionic particle number with respect to the original result. Since we are interested in regions of the bubble wall where sphalerons are still active, $i . e$ for values of $v_{i}(x) / T$ typically smaller than one, then the above mentioned suppression factor might be crucial.

The ultimate reason why these suppressions do not appear in the original treatment, is that considering eq. (国) as the only effect of the background is equivalent to perturbing around the Higgs field configuration $\delta(x)=0, v_{i}(x) \neq 0$, which is not a solution of the field equations. In other words, it is equivalent to disentangling $\delta(x)$ from $v_{i}(x)$, whereas from the field equations one can see that $\partial_{\mu} \delta(x)$ vanishes as $v_{i}(x)^{2}$ for vanishing $v_{i}(x)$.

The purpose of this letter is to compute the averages $n_{i}$ on the bubble wall background, both for fermions and Higgses, making use of a field theoretical approach. In this way we are able to treat the background consistently and to recover the expected suppression factors. We wish to stress that what we call here $n_{i}$ are just the perturbations induced by the $\mathrm{CP}$ violating background. They should be used as source terms for the departure from equilibrium in the equations describing dynamical processes, like gauge and Yukawa interactions, baryon number violation and particle diffusion.

Our starting point is the finite temperature generating functional for the 1PI Green's func- 
tions with insertion of an operator $\hat{O}(z)$ (in the following $\hat{O}(z)$ will represent a particle current)

$$
\Gamma\left[\Phi_{i}^{c}(x), \Delta(x)\right]=W\left[J_{i}(x), \Delta(x)\right]-\sum_{j} \int d^{4} x J_{j}(x) \Phi_{j}^{c}(x),
$$

where $\Phi_{i}^{c}(x)$ are the classical fields of the theory and $J_{i}(x)$ the corresponding sources, while $\Delta(x)$ is the source for the operator $\hat{O}(x)$.

The quantity we are interested in is the expectation value of the operator $\hat{O}(z)$ on the background given by the fields $\Phi_{i}^{c}(x)$, which we will specify later,

$$
\langle\hat{O}(z)\rangle_{\Phi_{i}^{c}(x)}=\left.\frac{1}{i} \frac{\delta \Gamma\left[\Phi_{i}^{c}, \Delta\right]}{\delta \Delta(z)}\right|_{\Delta=0} \equiv \mathcal{O}\left[\Phi_{i}^{c}(x)\right](z) .
$$

We can expand the functional $\mathcal{O}\left[\Phi_{i}^{c}(x)\right](z)$ in a power series of $\Phi_{i}^{c}$

$$
\mathcal{O}\left[\Phi_{i}^{c}(x)\right](z)=\sum_{n=0}^{\infty} \sum_{i_{1}, \ldots, i_{n}} \frac{1}{n !} \int d^{4} x_{1} \cdots d^{4} x_{n} \mathcal{O}_{i_{1}, \ldots, i_{n}}^{(n)}\left(x_{1}, \cdots, x_{n} ; z\right) \Phi_{i_{1}}^{c}\left(x_{1}\right) \cdots \Phi_{i_{n}}^{c}\left(x_{n}\right),
$$

where the coefficients of the expansion are the n-point 1PI Green's functions with one insertion of the operator $\hat{O}(z)$ computed in the unbroken phase

$$
\mathcal{O}_{i_{1}, \ldots, i_{n}}^{(n)}\left(x_{1}, \cdots, x_{n} ; z\right)=\left.\frac{1}{i} \frac{\delta^{n+1} \Gamma\left[\Phi_{i}^{c}, \Delta\right]}{\delta \Phi_{i_{1}}^{c}\left(x_{1}\right) \cdots \delta \Phi_{i_{n}}^{c}\left(x_{n}\right) \delta \Delta(z)}\right|_{\Phi_{i}^{c}=\Delta=0} .
$$

The relevant background $\bar{\Phi}_{i}^{c}(x)$ for us is given by the bubble wall, which is a solution of the field equations of motions

$$
\left.\frac{\delta \Gamma\left[\Phi_{i}^{c}, \Delta=0\right]}{\delta \Phi_{i}^{c}(x)}\right|_{\Phi_{i}^{c}=\bar{\Phi}_{i}^{c}}=0
$$

with appropriate boundary conditions.

In order to be more specific, we consider the extension of the standard model with two Higgs doublets where the up and down type quarks couple to different Higgs doublets. We consider here the case of spontaneous CP violation like that considered in ref. [8] for the MSSM.

The most general tree level effective potential is given by

$$
\begin{aligned}
V & =m_{1}{ }^{2}\left|H_{1}\right|^{2}+m_{2}{ }^{2}\left|H_{2}\right|^{2}-\left(m_{3}{ }^{2} H_{1} H_{2}+\text { h.c. }\right)+\lambda_{1}\left|H_{1}\right|^{4}+\lambda_{2}\left|H_{2}\right|^{4} \\
& +\lambda_{3}\left|H_{1}\right|^{2}\left|H_{2}\right|^{2}+\lambda_{4}\left|H_{1} H_{2}\right|^{2}+\left[\lambda_{5}\left(H_{1} H_{2}\right)^{2}+\lambda_{6}\left|H_{1}\right|^{2} H_{1} H_{2}+\lambda_{7}\left|H_{2}\right|^{2} H_{1} H_{2}+\text { h.c. }\right],
\end{aligned}
$$

where all the couplings are now assumed to be real. Note that the potential $V$ depends only on the phase $\delta=\theta_{1}+\theta_{2}$, whereas the orthogonal combination represents the gauge phase.

We assume that the parameters of the Lagrangian and the temperature are such that, when the loop corrections are considered, the potential assumes a double well shape and a expanding bubble solution exists for the complete equation of motion given by eq. (6). 
Since the interesting dynamics for baryogenesis takes place in a region close to or inside the bubble wall, we approximate it with an infinite plane travelling at a constant speed $v_{w}$ along the $z$ axis.

Considering eq. (6) at the tree level and including the one loop corrections to the effective potential, one can see that a solution exists for which all the background fields are vanishing except for the neutral Higgses. Their asymptotic behaviour is such that $v_{i}(z) \rightarrow 0$ for $z \rightarrow-\infty$ and $v_{i}(z) \rightarrow v_{i}^{+}$for $z \rightarrow+\infty$, where $v_{i}^{+}$are the finite temperature values for the Higgs fields in the broken phase. Moreover, due to the dependence on the phase $\delta$ of the potential, the two Higgs phases $\theta_{1,2}$ are space-time dependent, and their evolution is such that

$$
v_{1}^{2}(z) \partial^{\mu} \theta_{1}(z)=v_{2}^{2}(z) \partial^{\mu} \theta_{2}(z)
$$

This solution is the starting point for our expansion in eq. (4), from which we will now see that a non zero contribution to the neutral Higgs currents already exists at the tree level, whereas the currents for fermions and charged Higgses appear as loop effects. The fact that we are not considering the complete background solution to eq. (6) will modify our results only at higher orders.

We first consider the left handed top current $J_{t_{L}}^{\mu}=\overline{t_{L}} \gamma^{\mu} t_{L}$. The first non-vanishing term of the expansion in eq. (1) appears quadratically in the Higgs fields background, i.e.

$$
\left\langle J_{t_{L}}^{\mu}(z)\right\rangle=\left.\frac{1}{2} \sum_{i, j} \int d^{4} x d^{4} y \frac{\delta^{3} \Gamma\left[\bar{\Phi}_{i}^{c}, \Delta_{\mu}\right]}{\delta \bar{\Phi}_{i}^{c}(x) \delta \bar{\Phi}_{j}^{c}(y) \delta \Delta_{\mu}(z)}\right|_{\bar{\Phi}_{i, j}^{c}=\Delta_{\mu}=0} \bar{\Phi}_{i}^{c}(x) \bar{\Phi}_{j}^{c}(y),
$$

where we take $\bar{\Phi}_{i}^{c}=\left\{H_{1}^{0}, H_{1}^{0^{*}}, H_{2}^{0}, H_{2}^{0^{*}}\right\}$ to be the bubble wall Higgs background. Since we are interested in computing the averages in regions where the sphalerons are still active, i.e. for $v_{i} / T<1$ [1], higher order contributions to the expansion (4) can be safely neglected.

The first non-vanishing contribution to eq. (9) appears only at one loop, and is given by

$$
\left\langle J_{t_{L}}^{\mu}(z)\right\rangle^{(1)}=i \frac{h_{t}^{2}}{2} \int d^{4} x d^{4} y \operatorname{Im}\left(H_{2}^{0}(x) H_{2}^{0}(y)^{*}\right) \mathcal{G}^{\mu}(x, y, z),
$$

where $h_{t}$ is the top Yukawa coupling and $\mathcal{G}^{\mu}(x, y, z)$ is the Green function corresponding to the diagram in Fig. 1. As we have already remarked, in computing this diagram one must use the propagators in the unbroken phase. Note that this contribution vanishes if the phase $\theta_{2}$ of the Higgs field $H_{2}^{0}$ is a constant. The scale of the external momenta $p$ are set by the spacetime variation of the phase, which, in the case of interest for us of thick bubble walls $\left(L_{w} \simeq\right.$ $(10-100) / T)$ [12], allows us to neglect contributions of order $p^{2} / T^{2}$ in the high temperature 


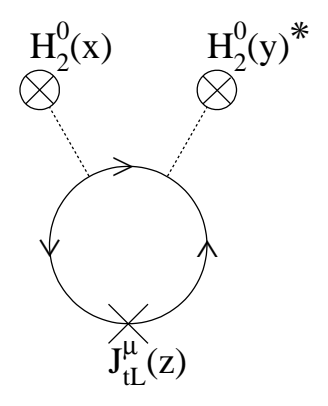

Figure 1: The 1-loop contribution to the left-handed top current.

expansion. Computing $\mathcal{G}^{\mu}(x, y, z)$ in the $\overline{M S}$ renormalization scheme at the scale $\mu$, we obtain, in the reference of frame of the thermal bath,

$$
\left\langle J_{t_{L}}^{\mu}(z)\right\rangle^{(1)} \simeq-\frac{h_{t}^{2}}{2 \pi^{2}} v_{2}^{2}(z) \partial^{\mu} \theta_{2}(z)\left(\log \left(\frac{\mu^{2}}{A_{f} T^{2}}\right)+\frac{7}{2}\right),
$$

where $A_{f}=\pi^{2} \exp \left(3 / 2-2 \gamma_{E}\right) \simeq 13.944$.

Eq. (11) shows the expected dependence on $h_{t}^{2}$ and $v_{2}^{2}(z)$ which, in comparison to the original result given in ref. [7], gives a suppression factor $\mathcal{O}\left(h_{t} v_{2} / \pi T\right)^{2}$.

A graph similar to that in Fig. 1 for the right handed top quark leads to a contribution to $\left\langle J_{t_{R}}^{\mu}(z)\right\rangle$ given by $\left\langle J_{t_{R}}^{\mu}(z)\right\rangle^{(1)}=-\left\langle J_{t_{L}}^{\mu}(z)\right\rangle^{(1)}$. For the other fermion species, one finds analogous results, in which $h_{t}$ is replaced by the appropriate Yukawa coupling, and $v_{2}(z)\left(v_{1}(z)\right)$ appears for the up (down)-type fermions.

A contribution to $\left\langle J_{t_{L}}^{\mu}(z)\right\rangle$ proportional to $\operatorname{Im}\left(H_{1}^{0} H_{2}^{0}\right)=v_{1} v_{2} \sin \delta$, and to $h_{t}^{2}$, appears at two loops, given by the graph in Fig. 2. Since the computation has to be performed in the unbroken phase, we must use resummed propagators for the Higgs fields in order to deal with the IR divergences [13].

In the unbroken phase the Higgs spectrum contains two complex neutral fields and two charged fields. The resummation can be achieved by considering the propagators for the eigenstates of the thermal mass matrix, which have masses given by

$$
M_{1,2}^{2}(T)=\frac{m_{1}^{2}(T)+m_{2}^{2}(T) \mp \sqrt{\left(m_{1}^{2}(T)-m_{2}^{2}(T)^{2}\right)^{2}+4 m_{3}^{4}(T)}}{2},
$$

where the $m_{i}^{2}(T)$ are the thermally corrected mass parameters of the potential (7), $m_{1}^{2}(T) \simeq$ $m_{1}^{2}+3 g^{2} T^{2} / 16, m_{2}^{2}(T) \simeq m_{2}^{2}+h_{t}^{2} T^{2} / 4$, while $m_{3}^{2}(T)$ receives only logarithmic corrections in $T$, which were computed in ref. [8]. 


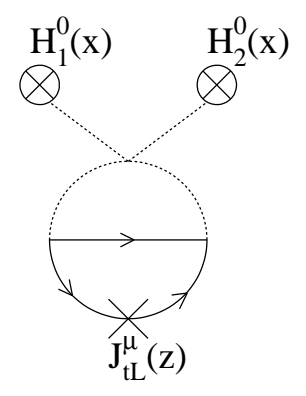

Figure 2: The 2-loop contribution to the left-handed top current. The scalar internal lines can be either neutral or charged Higgs fields.

Assuming $p^{2} \ll M_{1,2}^{2}(T) \ll(2 \pi T)^{2}$, where $p$ is again the external momentum $p \simeq 1 / L_{w}$, it is straightforward to see that the non-zero Matsubara modes of the bosonic integral are strongly suppressed. Keeping only the zero mode, we obtain

$$
\left\langle J_{t_{L}}^{\mu}(z)\right\rangle^{(2)} \simeq-\frac{\lambda_{5} h_{t}^{2}}{384 \pi^{3}} \frac{T m_{3}^{2}(T)}{\left(M_{1}(T)+M_{2}(T)\right)^{3}}\left(\log \left(\frac{\mu^{2}}{A_{f} T^{2}}\right)+\frac{7}{2}\right) \partial^{\mu}\left[v_{1}(z) v_{2}(z) \sin \delta(z)\right] .
$$

As for the one-loop result, the two loop contribution with neutral Higgses in the internal lines for $\left\langle J_{t_{R}}^{\mu}(z)\right\rangle$ is opposite to the one for $\left\langle J_{t_{L}}^{\mu}(z)\right\rangle$. This is no longer true when the graphs with charged Higgses in the internal lines are taken into account. In such a case, $\left\langle J_{t_{R}}^{\mu}(z)\right\rangle$ gets a a contribution equal to eq. (13), whereas the result for $\left\langle J_{t_{L}}^{\mu}(z)\right\rangle$ is analogous but proportional to $h_{b}^{2}$ instead of $h_{t}^{2}$. The charged Higgs loops give also rise to a non-vanishing left handed bottom density opposite to eq. (13). As we will discuss in the following, this fact may have interesting implications for the spontaneous baryogenesis mechanism.

On the background of the $\mathrm{CP}$ violating bubble wall non-vanishing Higgs currents $J_{H_{i}}^{\mu}=i\left(H_{i}^{\dagger} \mathcal{D}^{\mu} H_{i}-\mathcal{D}^{\mu} H_{i}^{\dagger} H_{i}\right)$, where $\mathcal{D}^{\mu}$ is the covariant derivative, are also present. In the case of the neutral Higgses a contribution already appears at the tree level, see Fig. 3, and is given by the classical current

$$
\left\langle J_{H_{i}^{0}}^{\mu}(z)\right\rangle^{(3)}=-2 v_{i}^{2}(z) \partial^{\mu} \theta_{i}(z) .
$$

Note that, again, the individual Higgs currents are zero if the phases are constant. Moreover, as one can easily see from the equation of motion (8), the total Higgs hypecharge current vanishes at the tree level.

At one loop, Fig. 4, we have a further contribution to the neutral Higgs current and the 


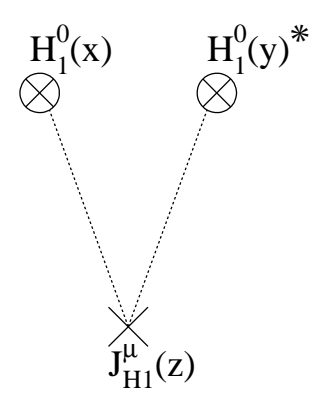

Figure 3: The tree level contribution to the neutral Higgs current.

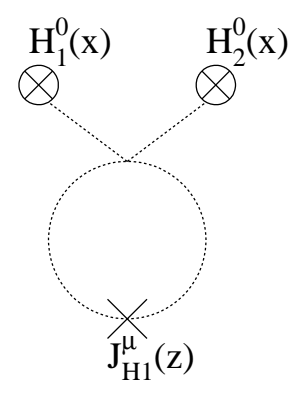

Figure 4: The 1-loop contribution to the neutral and charged Higgs currents.

first non-vanishing one to the charged Higgs current. For the neutral Higgs currents we obtain

$$
\left\langle J_{H_{1}^{0}}^{\mu}(z)\right\rangle^{(4)}=\left\langle J_{H_{2}^{0}}^{\mu}(z)\right\rangle^{(4)} \simeq \frac{\lambda_{5}}{8 \pi} \frac{T m_{3}^{2}(T)}{\left(M_{1}(T)+M_{2}(T)\right)^{3}} \partial^{\mu}\left[v_{1}(z) v_{2}(z) \sin \delta(z)\right],
$$

Each charged Higgs gets a contribution equal to that of the neutral Higgs belonging to the same doublet. As expected, also the Higgs currents vanish in the limit of vanishing $v_{i}(z)$.

In summary, we have made use of a field theoretical approach based on a expansion in the background fields around the unbroken phase to compute in a consistent way the perturbations to the particle currents induced by a $\mathrm{CP}$ violating bubble wall background. We have shown that the various contributions arise at the tree level, in the case of the Higgs currents, or as loop effects. In this way it has been possible to avoid the various ambiguities inherent to the traditional approach based on the rotation of the fields, recovering all the expected suppression factors and including the Higgs fields.

In order to get a feeling of the implications of these results for the spontaneous baryogenesis mechanism, we can first consider an adiabatic approximation similar to that discussed in ref. 
[14]. Inside the bubble walls the $(B+L)$-violating sphaleron transitions are biased since a non-zero local equilibrium value for $(B+L)$ is induced. $(B+L)_{E Q}$ is a linear combination of the expectation values of all the currents conserved by the interactions in equilibrium inside the bubble wall. Assuming that gauge flavor diagonal, top Yukawa and Higgs-Higgs interactions are in equilibrium 2 , the conserved charges are $Q^{\prime},(B-L)^{\prime}, B P^{\prime}=B_{3}-1 / 2\left(B_{1}+B_{2}\right)$ and $\tilde{Y}^{\prime}=Y_{H}+Y_{t_{L}}+Y_{t_{R}}+Y_{b_{L}}+1 / 3 Y_{l e p}^{\prime}$ where the prime means that only particles in equilibrium must be considered, and $Y_{l e p}^{\prime}$ is the hypercharge of the leptons in equilibrium. The values of these conserved currents are made up by the background perturbations computed above. Neglecting the contributions to the fermionic currents not proportional to $h_{t}^{2}$, it is straightforward to see that the contributions to $(B+L)_{E Q}$ from the two Higgs currents cancel each other both at the tree level and at one loop, and among the conserved charges only $\tilde{Y}^{\prime}$ gets a non-vanishing contribution at one loop. The two loop diagrams where charged Higgses are exchanged give a further contribution to $Q^{\prime}$ and $\tilde{Y}^{\prime}$.

The local equilibrium value $(B+L)_{E Q}$ enters a rate equation of the form $\dot{B} \simeq-\Gamma_{S P}(B+L)_{E Q} / T^{3}$ where $\Gamma_{S P}$ is the rate of the sphaleronic transition, describing the generation of baryon number inside the bubble walls. As a consequence, the force driving baryon number violation is just a one loop effect.

Regarding the final value for the baryon asymmetry in the adiabatic approximation, the suppression terms that we have found would give rise to a suppression factor $\mathcal{O}\left(h_{t}^{2} v_{c o}^{2} / \pi^{2} T^{2}\right)$ where $v_{c o}$ is the value of the Higgs fields for which the sphaleron transitions cease to be effective. Following ref. [11] and taking $v_{c o} / T \simeq \alpha_{w} / g$ we see that typically a suppression $\mathcal{O}\left(10^{-4}\right)$ arises. In this case, it might be hard to reconcile the observed value for the baryon asymmetry with this mechanism for baryogenesis in the adiabatic limit, both in the case of spontaneous and of explicit CP violation in the Higgs sector.

Nevertheless, it has been recently shown that particle diffusion may play an important role in the description of the spontaneous baryogenesis mechanism. As a consequence of the perturbation induced by the bubble wall background, and of the different diffusion coefficients of different particle species, asymmetric densities are formed not only inside the bubble walls, as in the adiabatic approximation, but also in front of it, where the sphaleron transitions are not suppressed. These asymmetries are then transformed into a baryon asymmetry mainly in the region in front of the bubble wall, in a scenario similar to that occurring in the case of thin bubble walls.

In order to improve the adiabatic approximation a system of kinetic equations describing

\footnotetext{
${ }^{2}$ In this approximation we also assume that the hypercharge violating processes whose rates are suppressed by powers of $v(T) / T$ are out of equilibrium.
} 
particle interactions and diffusion should then be solved, along the way of, e.g., ref. [9]. In this case, the quantities that we have computed would represent the sources of the departure from thermal equilibrium. This task goes beyond the scope of this letter, however an important, and maybe helpful, difference with respect to the situation considered in ref. [9] may be pointed out. As we have already discussed, in that paper the perturbation to the i-th particle species was simply proportional to $y^{i} \partial^{\mu} \delta T^{2}$, where $y^{i}$ is the hypercharge of the particle. This proportionality was due to the fact that a hypercharge rotation has been made to remove the Higgs phase from the Yukawa couplings. Such a dependence does not appear in our results, neither for the fermions nor for the Higgses. Instead, as long as the loops with charged Higgses are neglected, we obtain that, e.g., $\left\langle J_{t_{L}}^{\mu}\right\rangle=-\left\langle J_{t_{R}}^{\mu}\right\rangle$. This behaviour reflects the fact that in the Yukawa couplings left and right handed quarks have opposite couplings to the imaginary part of the Higgs field. When charged Higgses are taken into account, the contribution to $\left\langle J_{t_{R}}^{\mu}\right\rangle$ at two loops is opposite to that to $\left\langle J_{b_{L}}^{\mu}\right\rangle$ while $\left\langle J_{t_{L}}^{\mu}\right\rangle$ and $\left\langle J_{b_{R}}^{\mu}\right\rangle$ get also opposite contributions, but only proportional to $h_{b}^{2}$. Concerning the Higgs currents, they are proportional to $\lambda_{5}$ instead of $h_{t}^{2}$ and moreover, from eq. (15) we see that they are the same for the two neutral Higgses, which have opposite hypercharges.

In ref. [9] it was noted that, since all the perturbations were proportional to the hypercharge (and to $\dot{\delta}$ ), the only processes which could be biassed were the hypercharge violating ones, which have rates suppressed by powers of $v_{i}(z)$. Due to the smallness of these rates on the outer edge of the bubble wall, this gave rise to an important suppression on the value of the fluxes of particles diffused in the unbroken phase. On the other hand, our results do not exhibit such proportionality, then also the (unsuppressed) hypercharge conserving processes are actually biassed. As a consequence, inserting our perturbation in the kinetic equations one might expect that in the unbroken phase fluxes of roughly the same magnitude than those of ref. [9] would be found. A numerical treatment of the problem using the results of this letter and properly treating baryon number violation in the unbroken phase would then be highly desirable in order to understand whether diffusion can really help in overcoming the pessimistic results of the adiabatic approximation.

It is a pleasure to thank W. Buchmüller, J.R. Espinosa, F. Feruglio, and J. Ignatius for useful discussions. 


\section{References}

[1] V.A. Kuzmin, V.A. Rubakov, and M.E. Shaposhnikov, Phys. Lett. B155, 36 (1985).

[2] For a review see A. Cohen, D. Kaplan, and A. Nelson, Ann. Rev. Nuc. Part. Sci. 43, 106 (1993).

[3] A. Cohen, D. Kaplan, and A. Nelson, Nucl. Phys. B373, 453 (1992), Phys. Lett. B294, 57 (1992).

[4] J.R. Espinosa, M. Quirós and F. Zwirner, Phys Lett. B314, 206 (1993); W. Buchmüller, Z. Fodor, T. Helbig and D. Walliser, Ann. Phys. 234, 260 (1994); Z. Fodor and A. Hebecker, Nucl. Phys. B432 127 (1994); W. Buchmüller, Z. Fodor and A. Hebecker, hep-ph 9502321.

[5] K. Kajantie, K. Rummukainen and M.E. Shaposhnikov, Nucl. Phys. B407 356 (1993); K. Farakos, K. Kajantie, K. Rummukainen and M.E. Shaposhnikov, Phys. Lett. B336 494 (1994); B Bunk, E.M. Ilgenfritz, J. Kripfganz and A Schiller, Nucl. Phys. B403 453 (1993); F. Csikor, Z. Fodor, J. Hein, K. Jansen, A. Jaster, and I. Montvay, Phys. Lett. B334 405 (1994).

[6] J.R. Espinosa, M. Quirós and F.Zwirner, Phys. Lett. B307, 106 (1993); A. Brignole, J.R. Espinosa, M. Quirós. and F. Zwirner, Phys. Lett. B324, 181 (1994).

[7] A. Cohen, D. Kaplan, and A. Nelson, Phys. Lett. B263, 86 (1991).

[8] D. Comelli and M. Pietroni, Phys. Lett. B306, 67 (1993); D. Comelli, M. Pietroni, and A. Riotto, Nucl. Phys. B412, 441 (1994); Phys. Rev. D50, 7703 (1994); Phys. Lett. B343, 207 (1995); J.R. Espinosa, J.M. Moreno, and M. Quirós, Phys. Lett. B319, 505 (1993).

[9] A. Cohen, D. Kaplan, and A. Nelson, Phys. Lett. B336, 41 (1994).

[10] S.A. Abel, W.N. Cottingham, and I.B. Whittingham, Nucl. Phys. B410, 173 (1993).

[11] M. Dine and S. Thomas, Phys. Lett. B328, 73 (1994).

[12] M. Dine, R. Leigh, P. Huet, A. Linde, and D. Linde, Phys. Rev. D46, 550 (1992).

[13] P. Arnold and O. Espinosa, Phys. Rev. D47, 3546 (1993).

[14] D. Comelli, M. Pietroni, and A. Riotto, Padova preprint DFPD-94-TH-39, hep$\mathrm{ph} / 9406369$. 